\title{
COVID-19 in HIV-infected individuals
}

\author{
Pathum Sookaromdee ${ }^{1 *}$ (1), Viroj Wiwanitkit² (B)
}

Dear Editor,

We would like to share ideas on the publication entitled "Potential impact of the COVID-19 in HIV-infected individuals: a systematic review" ${ }^{1}$. de Medeiros et al. ${ }^{1}$ noted that "HIV did not show any relevance directly with the occurrence of COVID-19. Some studies suggest ... to control HIV infection could be used to prevent COVID-19 infection"1. In fact, the clinical association between COVID-19 and HIV infection is interesting. According to the pathobiological process of both infections, there is no common pathway regarding receptor and target cell. Hence, there should be no direct clinical association. Regarding the effect of antiviral therapy, the common antiviral activity of some antiviral drugs might be useful for the management of COVID-19. However, the dose adjustment might be required ${ }^{2}$. Nevertheless, the common clinical

\section{REFERENCES}

1. de Medeiros KS, da Silva LAS, Macêdo LTA, Sarmento AC, Costa APF, Eleutério J Jr, et al. Potential impact of the COVID-19 in HIV-infected individuals: a systematic review Impact of the COVID-19 in HIV-Infected Individuals. Rev Assoc Med Bras. (1992). 2021;67Suppl 1(Suppl 1):127-56. https://doi.org/10.1590/18069282.67.Suppl1.20200754 and geographical epidemiology of these two diseases should be discussed. In many developing countries, social determinants of health of HIV cases are usually poor and might support getting COVID-193.

\section{AUTHORS' CONTRIBUTIONS}

PS: Conceptualization, Data curation, Formal analysis, Funding acquisition, Investigation, Methodology, Project administration, Resources, Software, Supervision, Validation, Visualization, Writing - original draft, Writing - review and editing. VW: Conceptualization, Data curation, Formal analysis, Funding acquisition, Investigation, Methodology, Project administration, Resources, Software, Supervision, Validation, Visualization, Writing-original draft, Writing - review and editing.

2. Yasri S, Wiwanitkit V. Dose prediction of lopinavir/ritonavir for 2019-novel coronavirus (2019-nCoV) infection based on mathematic modeling. Asian Pac J Trop Med. 2020;13(3):137-8. https://doi. org/10.4103/1995-7645.277815

3. Brown LB, Spinelli MA, Gandhi M. The interplay between HIV and COVID-19: summary of the data and responses to date. Curr Opin HIV AIDS. 2021;16(1):63-73. https://doi.org/10.1097/ $\mathrm{COH} .0000000000000659$ 